\title{
高层大气建模: 从地球到行星
}

\author{
任志鹏 $1,2,3,4$
}

1. 中国科学院地质与地球物理研究所, 中国科学院地球与行星物理重点实验室, 北京 100029;

2. 中国科学院地质与地球物理研究所北京空间环境国家野外科学观测研究站, 北京 100029 ;

3. 中国科学院地球科学研究院, 北京 100029 ;

4. 中国科学院大学地球与行星科学学院, 北京 100049

E-mail: zpren@mail.iggcas.ac.cn

2019-12-09 收稿, 2020-01-18 修回, 2020-01-20 接受, 2020-04-08 网络版发表

中国科学院A类战略性先导科技专项(XDA17010404, XDA17010201)和国家自然科学基金(41874179, 41674158)资助

摘要地球和行星的高层大气作为大气圈与空间环境的过渡区, 是大气科学和空间科学的交叉研究领域. 高层大 气理论模式及其数值模拟可以突破实验观测的限制, 又能够比理论分析更为全面细致地重现高层大气的各种物理 和化学过程, 在高层大气的研究中表现出独特的优势, 也一直是国际高层大气研究热点之一. 虽然地球高层大气和 其他行星高层大气存在差异, 但它们之间有着密切的联系, 因此大多数的行星高层大气理论模式都是以地球高层 大气理论模式为基础开发的. 本文聚焦于地球和行星高层大气理论模式, 对目前国内外主流的地球和行星高层大 气理论模式的发展历史及其性能进行了系统介绍.

关键词高层大气, 大气环流模型, 电离层, 空间环境, 行星, 鸿鹄专项

地球的高层大气一般是指从海拔 $85 \mathrm{~km}$ 左右的中 间层顶到约 $1000 \mathrm{~km}$ 高度之间的高空大气区域. 高层大 气不仅包含有中性大气成分，还包含一定量由中性大 气电离而成的等离子体成分, 传统学界习惯把高层大 气的中性部分称为热层, 而把其中的电离成分称为电 离层. 在高层大气区域内, 电离层与热层相互作用、相 互耦合, 形成了一个复杂的耦合统一体, 因此也可以称 高层大气为电离层与热层耦合系统. 作为中性成分与 等离子体的复合体，高层大气与其他大气圈层存在明 显差异.

高层大气不但保护着地球不受太阳辐射和高能粒 子的破坏，同时还是人类进行无线电通信和探索太空 的重要区域. 在航天时代, 高层大气作为人类空间活动 的主要环境更是有着特殊的重要性. 电离层对无线通 信和GNSS导航定位有显著影响; 绝大部分的空间飞行
器(低轨航天器), 如空间站、资源遥感卫星、太阳同步 气象卫星和航天飞机等, 都基本运行在高层大气区域. 因此这些飞行器的寿命和轨道稳定性都依赖于热层中 性大气密度及其变化. 太阳活动、地磁活动都能够引 起高层大气的剧烈扰动(如电离层暴/热层暴等), 将严 重影响人类对空间环境的利用，对航天活动、地面技 术系统产生巨大影响. 因此我们需要对高层大气的变 化特征进行研究.

探测是研究高层大气的重要手段. 利用电离层垂 直测高仪、流星雷达、相干散射雷达、非相干散射雷 达、卫星和火箭等探测手段进行的地球和其他行星高 层大气(电离层和热层)观测已取得了大量成果, 极大地 丰富了我们对高层大气的认识. 然而, 由于观测在参数 覆盖性和时空覆盖性方面的限制，观测并不能完全解 决高层大气(特别是其他行星的高层大气)研究中所遇

引用格式: 任志鹏. 高层大气建模: 从地球到行星. 科学通报, 2020, 65: 1320-1335

Ren Z P. Upper atmosphere modeling: From Earth to Planet (in Chinese). Chin Sci Bull, 2020, 65: 1320-1335, doi: 10.1360/TB-2019-0803 
到的各种问题. 进一步深人研究高层大气系统中各种 相互作用过程的特性，揭示该系统中各种现象的形成 机理, 是学科发展的亟需, 也是空间物理学和空间天气 研究中一个极富挑战性的难题. 解决这一难题, 有赖于 对高层大气的实验观测、理论分析和数值模拟等综合 研究工作. 其中, 高层大气数值模拟以及作为其基础的 高层大气理论模式可以突破实验观测的限制，又能够 比理论分析更为全面细致地重现高层大气的各种物理 和化学过程, 在高层大气的研究中具有独特的优势, 一 直起着重要作用. 目前, 各种高层大气理论模式已成为 定量分析研究高层大气系统结构和动力学过程不可缺 少的重要手段.

\section{1 高层大气特性}

高层大气自身的物理和化学特性是高层大气建模 的基础, 也直接决定了高层大气模型与中低层大气模 型间的异同. 由于受到太阳紫外加热的影响, 高层大气 (热层)通常是大气层最热的区域(如地球的高层大气白 天通常能达到 $1000^{\circ} \mathrm{C}$ 以上), 该区域中性温度随高度增 加而升高, 在达到一定高度后(地球约为 $300 \mathrm{~km}$ )基本不 再随高度变化. 高层大气的基本结构和变化特征受到 一系列外部能量/动量源的控制，如太阳EUV和UV辐 射、极区高能粒子沉降、磁层等离子体对流、由中低 层大气上传到高层大气的各种大气波动等. 这些外部 能量/动量源会对高层大气进行加热，造成高层大气内 各种成分的电离和分解，进而驱动高层大气的全球风 场, 改变高层大气的中性和等离子体成分. 高层大气表 现出很多与中低层大气类似的性质. 如, 其密度在重力 的作用下随着高度的增加而指数衰减，形成垂直分层 结构. 但也表现出很多与中低层大气迥异的性质, 很多 在中低层大气中成立的物理近似会在高层大气中失效. 如，热层的强烈的昼夜温度变化驱动了热层巨大的水 平压力梯度，破坏了在中低层大气普遍适用的地转风 近似, 使得压力梯度力成为热层风场主要的驱动力, 并 进而驱动了强烈的热层水平风场.

高层大气与中低层大气的一个重要差异是大气化 学成分及其变化规律的差异. 以地球为例, 在湍流混合 作用较强的地球中低层大气区域，大气处于均匀混合 态, 大气的主要化学成分为氮气分子和氧气分子, 且其 相对含量基本不随高度变化. 而在地球热层高度, 由于 湍流混合作用随着高度的增加而持续削弱，地球热层 大气的中性化学成分在重力和扩散作用下也形成了明
显的垂直分层结构, 会随着高度发生明显的变化. 在地 球湍流层顶(地球约为 $110 \mathrm{~km}$ ) 以下湍流混合作用较强 的热层底部，中性大气的主要成分与地球中低层大气 相同，都是氮气分子和氧气分子，而氧气分子在太阳 $\mathrm{UV}$ 辐射的光化学作用下分解形成的氧原子的含量也 随着高度增加而逐步升高. 在 $110 \mathrm{~km}$ 以上, 湍流混合作 用相对削弱，中性大气的各种不同成分在重力和扩散 作用下根据各自的分子量开始逐步分离，氧原子逐步 成为主要的地球热层大气成分，并在约 $250 \mathrm{~km}$ 以上高 度成为主导性的地球中性大气化学成分. 而在更高的 高度, 氦原子和氢原子开始占据主导. 图1为MSIS热层 经验模型给出的一个典型的地球赤道白天的不同中性 化学成分大气密度的平均剖面. 热层大气化学成分的 高度变化引起了一系列与成分相关的物理量也表现出 明显的高度变化. 如, 在中低层大气中近似为常量的大 气比热容, 在热层高度不再是常量, 而会随着大气化学 成分的变化而变化.

高层大气与中低层大气的另一个主要差异就是其 中等离子体(电离层)的存在. 由于太阳极紫外线和X射 线以及源于磁层的高能沉降粒子的作用, 中高层大气 会部分电离，形成由大量正离子、负离子和自由电子 组成的电离层. 与中层大气重叠的电离层部分通常密 度很低, 对中层大气过程几乎没有影响. 但是，电离层 和热层都是高层大气的主要部分，并通过高层大气系 统内的化学过程、动力学过程、热力学过程和电动力 学过程等紧密耦合在一起.

(1) 在化学过程方面. 热层中性成分作为主要的电

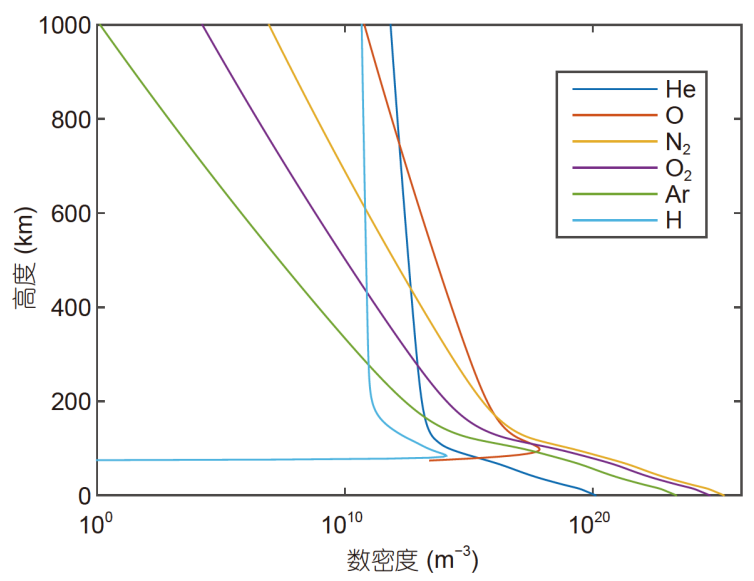

图 1 典型的地球赤道白天的不同中性化学成分大气密度的平均 剖面

Figure 1 The altitudinal profiles of atmospheric density of different neutral chemical components 
离源, 受到太阳辐射和高能粒子的作用而被电离, 进而 形成电离层中的离子和电子, 不但从源头决定了电离 层等离子体的成分和产生率，同时中性成分还通过参 与化学反应进一步调节电离层离子密度和离子成分; 而电离成分通过化学反应与中性成分相互作用，也通 过影响中性成分的化学循环过程, 改变了热层中性大 气的成分和密度.

(2) 在热力学过程方面. 离子和电子的大部分冷却 机制都受到中性成分的调节, 而它们的加热机制也受 到中性背景的影响; 离子化学反应放热和中性成分在 与电子/离子碰撞过程中伴随的能量吸收也是热层中性 大气极为重要的热源; 特别是, 高层大气吸收外部(太 阳、磁层等)能量的过程, 通常都必须是电离层和热层 同时参与才能完成.

(3) 在动力学过程方面. 中性成分和离子通过碰撞 相互影响了对方的动力学特征; 中性风的运动驱动了 等离子体的场向运动, 进而改变了等离子体动力学和 密度分布; 而离子通过在平行和垂直于磁场的方向上 对中性成分施加离子曳力, 影响中性动力学, 并成为热 层中性风场的主控因素之一.

(4) 在电动力学过程(如地球的电离层发电机过程) 方面. 地球的电离层发电机过程通过激发电离层电场, 显著影响了地球电离层/热层系统的电动力学过程, 地 球中低纬电离层电场就直接受控于电离层发电机效应, 而地球电离层发电机是地球高层大气中性风、中性成 分、电离成分和地磁场通过相互作用共同驱动的; 电 离层电场直接驱动了等离子体漂移, 影响了等离子体 密度分布, 并能够激发电离层不规则体; 电离层电场也 通过焦耳加热和离子曳力直接影响了热层中性成分的 温度、密度、成分和风场; 电离层发电机过程还通过 沿磁力线的映射过程, 把高层大气底部和上部联系起 来, 实现了高层大气内部能量和动量的垂直传输.

总而言之, 电离层和热层通过高层大气系统内一 系列复杂的物理和化学过程相互作用、相互影响, 紧 密耦合在一起.

高层大气还同相邻的中间层大气和磁层相互作 用、相互影响, 耦合在一起. 中间层大气主要通过两条 途径影响电离层/热层系统. 一方面, 通过发生在中间 层-低热层区(mesosphere-lower thermosphere, MLT, 地 球的相应高度为 $60 \sim 150 \mathrm{~km}$ ) 的湍流扩散等过程, 中层 大气的能量、动量和物质进人到高层大气内部. 另一 方面, 从中层大气传导到高层大气的各种大气波动, 如
各种频率的大气潮汐和行星波等, 也在中低层大气与 高层大气的耦合过程中扮演了重要角色. 首先, 这些波 动控制了低热层中性风场, 进而通过影响地球电离层 $\mathrm{E}$ 区(与低热层区域基本重合)发电机过程调节了电离层 电场, 调节后的电场沿磁力线映射到电离层/热层的各 个高度, 进而显著影响了整个高层大气的动力学过程. 其次, 上传到低热层高度的这些大气波动通常会在低 热层发生破碎和耗散. 在这个过程中, 它们会释放出自 身携带的动量和能量, 加速和加热低热层, 并显著影响 了该区域附近大气的混合与扩散。这些机制不但显著 改变了低热层的温度、成分和风场, 而且通过热层内 部的各种扩散机制和黏滞作用进一步影响了整个高层 大气系统, 实现了中层大气与高层大气的耦合. 通过场 向电流、对流电场和粒子沉降等过程, 高层大气也和 相邻的磁层发生了密切地耦合. 使磁层能量和动量以 地磁高纬区为进人高层大气系统的主要通道, 源于磁 层的高纬电离层电场和极光高能粒子沉降在高层大气 与磁层的耦合过程中扮演了重要角色. 这一过程受到 太阳风与行星际磁场的直接影响, 是整个日地空间环 境耦合链中的重要一环.

\section{2 地球高层大气建模研究历史}

高层大气的以上特性直接决定了, 高层大气不能 简单视为中低层大气的延伸, 高层大气模型也不能直 接由中低层大气模型改进而来. 高层大气理论模式在 模型设计、坐标系选择和模型算法都必须考虑到高层 大气特性带来的种种限制. 因此, 高层大气理论模式经 历了长期发展才逐步完备起来. 下面简介国际上高层 大气理论模式的发展历史, 并介绍目前主流的高层大 气理论模式.

早在 20 世纪60年代, 科学家们就开始研究高层大 气的理论建模问题. 学界首先采用观测数据或经验模 型所描述的热层三维压强场或温度场, 进而推导其他 物理量的变化趋势. 如, 通过采用给定的压强分布作为 动量源, Kohl和King ${ }^{[1]}$ 建立了最早的全球热层模式, 并 证实了太阳EUV辐射驱动的热层风的存在. 然而, 这类 高层大气模式受到所使用的观测数据或经验模型的限 制, 无法完全做到内部的逻辑自洽, 虽然在高层大气研 究中起到了重要作用但是一直没能成为高层大气模式 的主流.

学界一直以发展更为自洽的高层大气理论模式为 主流. 由于计算条件的限制, 早期自洽的高层大气理论 
模式一般都是二维模式，只能模拟稳态的高层大气场 (如风场和温度场). 然而, 由于无法有效估算高层大气 的纬向动量源项等关键驱动项，二维模式无法很好地 模拟高层大气的地方时变化、对外界能量注人的响应 特征(如高层大气对磁暴的响应)等核心时空变化特征. 此外，为了克服观测支撑的不足和实现模式的稳定运 行，早期的二维模式经常采用基于猜测的(通常包含一 定错误的)驱动项，且简化甚至忽略方程中的一些重要 项(如科里奥利力项等), 从而严重限制了早期的研究成 果的适用性. 但早期的二维模式所取得了系列成果, 为 对后来三维时变模式的发展起到了重要的指导作用.

自20世纪70年代开始，学界开始采用有限差分法 来发展自洽的三维时变的高层大气理论模式. 然而, 由 于计算条件的限制，早期的三维模式多通过假定高层 大气的水平结构或假定模式具有周期解，从而把模式 简化为一个一维垂直坐标系下的微分方程来求解. 如, Lindzent和Hong ${ }^{[2]}$ 就基于经典潮汐理论采用该方式构 建了一个高层大气潮汐理论模式. 这种研究方式虽然 取得了部分研究成果，但也严重限制了该类模式的应 用范围. 在 20 纪 70 年代末，随着计算机技术的不断发 展，开发真正的三维时变的高层大气理论模式(也称为 热层大气环流模式)才成为可能, 科学家们在原有二维 模式的基础上，开始发展三维时变的高层大气理论模 式. 如Fuller-Rowell和Rees ${ }^{[3]}$ 及Dickinson等人 ${ }^{[4]}$ 就分别 独立地发展了三维时变的热层模式. 后来, 他们的模式 被进一步发展为CTIPe(coupled thermosphere ionosphere plasmasphere electrodynamics model)和TIEGCM 这两个目前国际上广泛认可的高层大气理论模式.

美国大气研究中心(NCAR)开发的TIEGCM模式 (thermosphere-ionosphere-electrodynamics general circulation model)是目前世界上最著名的、应用最广泛的 高层大气理论模式. 该模式是从NCAR早期开发的二 维高层大气理论模式发端, 经过近 20 年的持续改进而 逐步发展起来的. Dickinson等人 ${ }^{[5,6]}$ 在 20 世纪70年代首 先利用有限差分法构建了一个二维高层大气理论模式. 在获得足够的计算条件后，Dickinson等人 ${ }^{[4]}$ 以这个二 维模式为基础, 通过给定适当的边界条件, 进一步发展 了一个三维热层大气环流模式TGCM(thermosphere general circulation model). 该模式采用压力坐标系, 在 垂直方向上把 90 500 km分为 24 个等压面来求解, 模式 的纬度-经度分辨率为 $5^{\circ} \times 5^{\circ}$. 通过自洽求解热层中性大 气的能量方程、动量方程和连续性方程, 该模式能够
模拟全球高层大气中性温度、压力和风场的分布. 该 模式基于有限差分法构建，为减小计算量在水平方向 上采用显格式差分，但是为保持模式稳定在垂直方向 上采用隐格式差分. 该版本TGCM的主要局限在于不 能自洽求解热层中性成分的分布，而是依赖高层大气 经验模型提供不同中性成分的百分比含量.

为解决高层大气中性化学成分自洽计算问题，基 于所提出的方法 ${ }^{[7]}$, Dickinson等人 ${ }^{[8]}$ 通过在模式中引人 不同大气化学成分的扩散/输运过程和化学产生/损失 过程，在TGCM模式中进一步实现了 3 种主要成分氧原 子 $\mathrm{O}$ 、氧气分子 $\mathrm{O}_{2}$ 和氮气分子 $\mathrm{N}_{2}$ 的质量百分比含量的 自洽求解，进一步完善了TGCM模式. 该版本TGCM缺 少自洽的电离层参数的计算, 而是由电离层经验模型 来提供模式运行所需的电子密度等电离层参量的分布. 该版本TGCM虽然考虑了中性氧元素的化学循环过 程、太阳EUV和UV辐射加热过程以及结合了高能粒 子沉降的极区焦耳加热过程 ${ }^{[9,10]}$, 但是对这些过程的模 拟并不完备和自洽, 导致其效果相对有限.

$\mathrm{TGCM}$ 是 NCAR 高层大气理论模式基础，基于 TGCM的一系列模拟研究表明，中性成分与等离子体 的相互作用是影响高层大气变化的重要因素，因而 $\mathrm{TGCM}$ 模式开始向热层-电离层耦合模式的方向发展， 为开发相应的热层-电离层耦合模式, Roble等人 ${ }^{[11]}$ 在 20 世纪70 80年代热层研究成果的基础上，首先构建了一 个一维的电离层-热层耦合模式, 用于模拟高层大气(电 离层和热层)的全球平均高度分布，评估不同的物理和 化学过程在其中的相对重要性. 该模式通过引人一系 列次要化学成分, 实现了较为完备的高层大气氧元素 和氮元素的化学循环过程和自洽的电离层与热层的加 热和冷却过程.

在这个一维模式研究成果的基础上，以TGCM热 层环流模式为基础，通过在每个时间步的每个格点上 都考虑热层与电离层的耦合, Roble等人 ${ }^{[12]}$ 建立了电离 层/热层耦合模式TIGCM(themrosphere-ionosphere general circulation model). TIGCM模式能够在地理坐标系 下耦合求解热层和电离层的非线性的动量方程、能量 方程、连续性方程、流体静力学方程和状态方程，自 洽计算一定地球物理条件下的地球高层大气(包括电 离层和热层)结构，计算给定氧原子 $\mathrm{O}$ 、氧气分子 $\mathrm{O}_{2}$ 、 氮气分子 $\mathrm{N}_{2} 、$ 一氧化氮分子 $N O 、 N\left({ }^{4} \mathrm{~S}\right)$ 和 $N\left({ }^{2} \mathrm{D}\right)$ 氮原 子、氦原子 $\mathrm{He}$ 、氩原子 $\mathrm{Ar}$ 和氧原子离子 $\mathrm{O}^{+}$、一氧化氮 分子离子 $\mathrm{NO}^{+}$、氧气分子离子 $\mathrm{O}_{2}{ }^{+}$、氮气分子离子 
$\mathrm{N}_{2}{ }^{+}$、氮原子离子 $\mathrm{N}^{+}$以及电子的数密度，电子、离子与 中性成分的温度及中性风场. 该模式考虑了地理极与 地磁极的分离, 引人了与极光过程相对应的极区高能 粒子沉降和磁层电场映射到高纬电离层后驱动的高纬 电离层环流，从而引人了磁层过程对高层大气的影响. 该模式通过在下边界的中性温度和中性风场中引人大 气潮汐 (主要包括周日潮汐和半日潮汐), 考虑了中低层 大气对高层大气的影响 ${ }^{[13]}$. TIGCM模式对于高层大气 (热层和电离层)结构的模拟结果与主流的热层和电离 层经验模型的结果基本一致，表明该模式已经成功包 括了热层和电离层中主要的大尺度物理和化学过程.

TIGCM的主要缺陷在于其控制中低纬电离层电动 力学过程的电离层电场是由经验模型提供的而不是自 洽计算的, 从而缺少了一个重要的电离层-热层耦合过 程. 因而, 在TIGCM模式的基础上, Richmond等人 ${ }^{[14]}$ 通 过引人基于地磁APEX坐标系的电离层发电机过程 ${ }^{[15]}$, 在模式内部实现了中低纬电离层电场的自洽计算, 发 展了TIEGCM模式(thermosphere-ionosphere-electrodynamics general circulation model). 该模式能够在非偶极 地磁场下运行, 通过自洽计算电离层发电机效应, 实现 了基于电离层发电机过程的电离层/热层耦合. 只要给 定高层大气的外部条件, 如太阳EUV和UV辐射通量、 极区极光粒子沉降通量、高纬电离层电场、从中低层 大气向上传播到高层大气的大气潮汐等, TIEGCM模式 就能够较好地再现热层和电离层的基本结构和变化特 征. 至此, TIEGCM这个国际上认可最广泛的高层大气 理论模式主体发展完成. 直到今天TIEGCM模式仍在不 断吸纳最新的高层大气研究成果, 但主要通过细节和 参数改进的方式进行迭代发展.

由于TIEGCM模式优异的性能, 学界在该模式基础 上还进一步研发了一系列衍生模式. 如, 为更好地研究 中低层大气对高层大气的影响, 学界基于TIEGCM研发 了中间层/热层/电离层/电动力学耦合模式TIMEGCM (thermosphere-ionosphere-mesosphere-electrodynamics general circulation model). 与TIGCM发展过程类似， Roble $^{[16]}$ 首先构建了一个一维的中间层-热层-电离层耦 合模式(模拟范围高度范围30 500 km), 用于模拟中高 层大气的全球平均高度分布, 评估不同的物理和化学 过程在其中的相对重要性. 该模式研究表明, 为较为全 面地模拟中间层-热层-电离层主要的化学循环过程, 需 要再引人臭氧 $\mathrm{O}_{3}$ 等一系列化学成分, 从而实现较为完 备的中高层大气氧元素、氮元素、氢元素和碳元素的
化学循环过程和自洽的中高层大气加热和冷却过程. 在此基础上, Roble和Ridley ${ }^{[17]}$ 将TIEGCM模式的下边 界从海拔 $97 \mathrm{~km}$ 向下延伸到 $30 \mathrm{~km}$, 在模式内引人了地 球平流层上部和中间层的物理、化学过程, 加人瑞利 摩擦和重力波参数化, 从而构建了中间层/热层/电离 层/电动力学耦合模式TIMEGCM. 该模式在许多有争 议的地球中间层和低热层大尺度问题研究和中低层大 气-高层大气耦合研究中都起到了重要作用. Wang等 人 $^{[18,19]}$ 则在TIGCM模式的基础上，通过嵌套网格的方 法成功提高了该模式的区域分辨率，从而开发了时空 高分辨率的TING模式(thermosphere ionosphere nested grid model).

此外, TIEGCM模式还通过与其他区域理论模式的 耦合实现了跨圈层的模拟. 如 20 世纪 90 年代, TIEGCM 模型就通过与IMM(ionosphere-magnetosphere model)磁 层模型结合, 初步实现了热层/电离层/磁层的耦合模 拟 ${ }^{[20]}$. 进人 21 世纪后, TIEGCM模型更通过与LFM (Lyon-Fedder-Mobarry global magnetosphere MHD code)磁层磁流体力学模型以及RCM(rice convection model)内磁层环流模型的结合，开发了耦合自洽的热 层/电离层/磁层耦合模式CMIT(coupled magnetosphereionosphere-thermosphere model ${ }^{[21]}$ ). Mendillo等人 ${ }^{[22]}$ 则 尝试把TIMEGCM模式与NCAR开发的通用气候学模 式CCM(community climiate mdoel)结合起来, 建立了一 个低层大气/中间层/热层/电离层的通量耦合模式: TIMEGCM-CCM3(thermosphere-ionosphere-lower atmosphere flux-coulped model). 这些理论模式都在跨圈 层耦合领域取得了重要的研究进展.

英国的 CTIP(coupled thermosphere-ionosphere-plasmasphere model)是目前国际上普遍认可的高层大气理 论模式之一. 该模式也是世界上第一个实现了自洽的 三维电离层/热层耦合模拟的高层大气理论模式. 20 世 纪80年代, Fuller-Rowell和Rees ${ }^{[3]} 、$ Rees等人 ${ }^{[23]}$ 在伦敦 大学学院(University College London, UCL)首先采用 有限差分法开发了作为CTIP前身的热层三维时变模式 GCM(general circulation model). GCM模式也采用压力 坐标系, 假定 $80 \mathrm{~km}$ 高度的地球大气是一个等压面, 并 把该等压面作为模式底边界, 然后将80 450 km的地球 高层大气垂直划分为 15 个等压面进行模拟. GCM模式 的纬度分辨率可根据模拟要求而改变, 但是其在经度 上固定将地球均匀划分为 20 个经度扇区(扇区间的间 隔为 $18^{\circ}$ ), 其时间步长为 $1 \mathrm{~min} . \mathrm{GCM}$ 模式考虑了地球曲 
率和科里奥利力的影响，在模式的垂直方向和水平方 向都考虑了黍滞项等非线性项的影响. 模式采用了包 括了水平压力梯度、水平与垂直黏滞力、离子曳力等 影响的非线性的动量方程, 也采用了能够描述内能、 动能及势能相互转化过程的包含了热传导、太阳UV 和EUV辐射加热、热层红外辐射冷却等加热/冷却过 程的非线性的能量方程，并实现了自洽的中性高层大 气的能量方程和动量方程求解. 此外, GCM模式还包 含了作为极区动量源和能量源的高纬电离层电场所驱 动的离子曳力和焦耳加热. 与该模式以前的模式相比, GCM 模式能够更好地模拟热层风场和温度场的三维时 变结构.

与早期的 TGCM模式类似，早期的GCM模式也不 能自洽求解热层中性成分的分布，而需要从经验模型 中获得高层大气不同中性成分的百分比含量. 因此, GCM模式建立后，UCL的科学家们对它进行了一系列 改进. Fuller-Rowell和Rees ${ }^{[24]}$ 在GCM模式中考虑了两 种主成分 (轻成分 $\mathrm{O}$ 和重成分 $\mathrm{O}_{2}$ 和 $\mathrm{N}_{2}$ ), 假定 $\mathrm{O}$ 和 $\mathrm{O}_{2}$ 处于 光化学平衡状态, 并成功描述了由热层动力学过程驱 动的两种主成分的输运过程以及由分子扩散和湍流扩 散所驱动的大气扩散过程，进而完成了热层平均中性 分子质量方程的推导, 实现了热层平均中性成分的自 洽求解. 值得一提的是, Fuller-Rowell和Rees所推导的 方程还被进一步应用于主要由两种气体构成的其他行 星大气模式的开发中.

通过改进原有的英国谢菲尔德大学的电离层模 式 ${ }^{[25,26]}$ 来开发电离层模块, Fuller-Rowell等人 ${ }^{[27]}$ 成功地 把UCL的GCM模式进一步发展成一个电离层/热层耦 合模式CTIM(coupled thermosphere- ionosphere model). 该模式将中低纬区域分为 20 个磁子午面，每个磁子午 面内选取 15 根磁通管，并同时求解这些磁通管中氧原 子离子 $\mathrm{O}^{+}$与氢原子离子 $\mathrm{H}^{+}$的数密度、离子场向运动速 度、离子温度和电子温度. 在每一时间步, 电离层模块 所需的中性背景大气参量和热层模块中所需的离子密 度、温度和速度等电离层参量都基于另一模块的同步 结果插值给出. CTIM模式包括了电离层E区和F区的化 学过程, 是研究高层大气内部中性-等离子体相互作用 的重要工具. Millward等人 ${ }^{[28]}$ 将CTIM模式继续向上扩 展，在模式中考虑了等离子体层对电离层和热层的影 响，通过构建同时运行并相互间双向耦合的全球热层 模块、基于开放磁力线的高纬电离层模块和基于闭合 磁力线的中低纬电离层/等离子体层模块, 建立了热层/
电离层/等离子体层耦合模式: CTIP(coupled themrosphere-ionosphere-plasmasphere model). 后来, CTIP模 式还实现了电离层电场的自洽计算, 形成了 CTIPe模式 (Millward等人 ${ }^{[29]}$ ). Harris等人 ${ }^{[30]}$ 则进一步将中间层和 平流层上部的物理、化学过程包括进CTIP中，形成了 CMAT模式(coupled middle atmosphere and thermosphere general circulation model).

Namgaladze等人 ${ }^{[31 \sim 33]}$ 也使用有限差分法发展了一 个自洽的地球热层/电离层/质子层耦合模式: GSM TIP (global self-consistent model of the thermosphere, ionosphere and protonosphere). 对于中性大气, 该模式采用 高度坐标系自洽计算了 $80 \sim 520 \mathrm{~km}$ 内的氧气分子 $\mathrm{O}_{2}$ 、 氮气分子 $\mathrm{N}_{2}$ 、氧原子 $\mathrm{O}$ 的数密度、中性温度和中性风 场. 对于地球电离层, 该模式基于光化学平衡计算地球 的 $\mathrm{D} 、 \mathrm{E}$ 和 $\mathrm{F}_{1}$ 层的等离子体密度, 而对电离层 $\mathrm{F} 2$ 层和质 子层则基于 $\mathrm{O}^{+} 、 \mathrm{H}^{+}$的连续性方程与能量方程求解得到 等离子体密度与温度. 通过求解 $\mathrm{E}$ 区电离层发电机方 程, 模式的电离层电场实现了自洽计算. 该模式的地磁 场使用中心偶极场, 并假定磁壳数 $L \geqslant 15$ 的磁力线为开 磁力线. 该模式一个重要特色是完全舍弃了地理坐标 系, 而直接在完全的地磁坐标系下进行数值模拟.

以往的高层大气理论模式都是基于静力平衡假设 构建, 从而简化模式的出发方程, 提高模式稳定性. Ridley等人 ${ }^{[34]}$ 开创性地放弃了垂直方向上的静力平衡假 设, 开发全新高层大气理论模式GITM(global ionosphere thermosphere model). 该模式在垂直方向上采用 高度网格, 在地理坐标系下耦合求解热层和电离层非 线性的动量方程、能量方程、连续方程、流体静力学 方程和状态方程，计算给出 $\mathrm{O} 、 \mathrm{O}_{2} 、 \mathrm{~N}_{2} 、 \mathrm{~N}\left({ }^{2} \mathrm{D}\right) 、 \mathrm{~N}$ $\left({ }^{2} \mathrm{P}\right) 、 \mathrm{~N}\left({ }^{4} \mathrm{~S}\right) 、 \mathrm{NO} 、 \mathrm{H} 、 \mathrm{He}$ 和 $\mathrm{O}\left({ }^{4} \mathrm{~S}\right)^{+} 、 \mathrm{O}\left({ }^{2} \mathrm{D}\right)^{+} 、 \mathrm{O}\left({ }^{2} \mathrm{P}\right)^{+} 、$ $\mathrm{O}_{2}^{+} 、 \mathrm{~N}_{2}^{+} 、 \mathrm{NO}^{+} 、 \mathrm{~N}^{+} 、 \mathrm{H}^{+} 、 \mathrm{He}^{+}$的数密度，电子、离子 与中性成分的温度和中性风速. 由于放弃了静力平衡 假设，该模式在模拟磁暴/亚暴期间的高层大气扰动状 态时获得了许多新发现, 表现出一定的优势.

在现有中低层大气和高层大气建模研究的基础上, 国际上也开始研发能从地面一直模拟到逃逸层顶的全 大气耦合模式，如日本的全大气耦合模式GAIA (ground-to-topside model of atmosphere and ionosphere for aeronomy ${ }^{[35]}$ )、美国NOAA的全大气耦合模式WAM (whole atmosphere model ${ }^{[36,37]}$ ) 和美国NCAR的全大气 耦合模式WACCM-X(whole atmosphere community climate model-eXtended ${ }^{[38,39]}$ ). 这些模式研究的核心目标 
是低层大气-中层大气-高层大气耦合等全大气系统耦 合问题, 高层大气模块在其中是作为组成部分存在.

在高层大气建模领域, 国内也进行了大量研究, 取 得了一系列进展. Wang和Xiao ${ }^{[40]}$ 通过对电离层/热层的 耦合特性进行了数值研究, 建立了一个二维电离层/热 层系统耦合模式, 并利用该模式对电离层/热层耦合进 行了研究. Lei等人 ${ }^{[41]}$ 则利用高层大气经验模型 MSIS00和IRI2000作为输人通过求解中性大气的 Navier-Stokes动量方程建立了一个三维的热层风场理论 模式. Ren等人 ${ }^{[42]}$ 发展了一个自洽的三维时变的全球电 离层/热层/电动力学耦合模式GCITEM-IGGCAS(global coupled ionosphere-thermosphere-electrodynamics model, Institute of Geology and Geophysics, Chinese Academy of Sciences).

GCITEM-IGGCAS模式能够模拟90 600 km之间 电离层/热层区域的大气物理和化学过程. 该模式通过 自洽求解电离层/热层的动量方程、能量方程、质量方 程、流体静力学方程和状态方程, 自洽计算90 600 km 之间电离层/热层区域的主要中性成分 $\mathrm{O} 、 \mathrm{O}_{2}$ 和 $\mathrm{N}_{2}$, 次 要中性成分 $\mathrm{N}\left({ }^{4} \mathrm{~S}\right) 、 \mathrm{~N}\left({ }^{2} \mathrm{D}\right) 、 \mathrm{NO} 、 \mathrm{Ar}$ 和He, 电离成分 $\mathrm{O}^{+} 、 \mathrm{~N}_{2}^{+} 、 \mathrm{O}_{2}^{+} 、 \mathrm{NO}^{+} 、 \mathrm{~N}^{+}$离子及电子的数密度, 电 子、离子和中性成分的温度以及中性风场的时变三维 结构. 该模式还能够通过调用TIDM-IGGCAS-II模式 ${ }^{[43]}$ (theoretical ionospheric dynamo model, Institute of Geology and Geophysics, Chinese Academy of Sciences,
Version II)自洽计算中低纬电离层电场(可直接换算为 等离子体电动力学漂移速度). GCITEM-IGGCAS基于 地理坐标系采用有限差分法求解, 其经度-纬度的网格 设为 $7.5^{\circ} \times 5^{\circ}$, 时间步长为 $2 \sim 5 \mathrm{~min}$. GCITEM-IGGCAS 模式基于静力平衡假设构建, 它在垂直方向上没有使 用很多电离层/热层耦合模式所通常使用的压力坐标, 而是使用了高度坐标. 该模式在垂直方向上的空间步 长会随着高度变化, 低热层高度的空间步长约为 $3 \mathrm{~km}$, 而高热层高度的空间步长可达到 $30 \mathrm{~km}$. 该模式较好地 再现了电离层/热层的基本形态和主要变化特征. 图2给 出了该模式的基本框架, 而图3则给出了该模式模拟结 果与经验模型对比的一个例子.

2018年3月 12 日, 中国科学院启动了一项 $\mathrm{A}$ 类战略 性先导科技专项——临近空间科学实验系统(简称“鸿 鹄专项”), 其科学目标是突破临近空间浮空器平台关键 技术, 开展临近空间科学研究. 临近空间大气(高度范围 约20 100 km) 与高层大气的相互耦合研究是“鸿鹄专 项”的核心科学问题之一, 由中国科学院地质与地球物 理研究所牵头研究. 为支持相关科学研究, 在“鸿鹄专 项”支持下, 中国科学院地质与地球物理研究所研究团 队正以GCITEM-IGGCAS模式为基础，通过把模式下 边界从 $90 \mathrm{~km}$ 向下延伸到 $30 \mathrm{~km}$, 在模式内引人了地球 平流层上部和中间层的相关物理、化学过程, 从而新 开发一个临近空间-高层大气耦合理论模式.

表 1 给出了目前主流的地球高层大气理论模式的

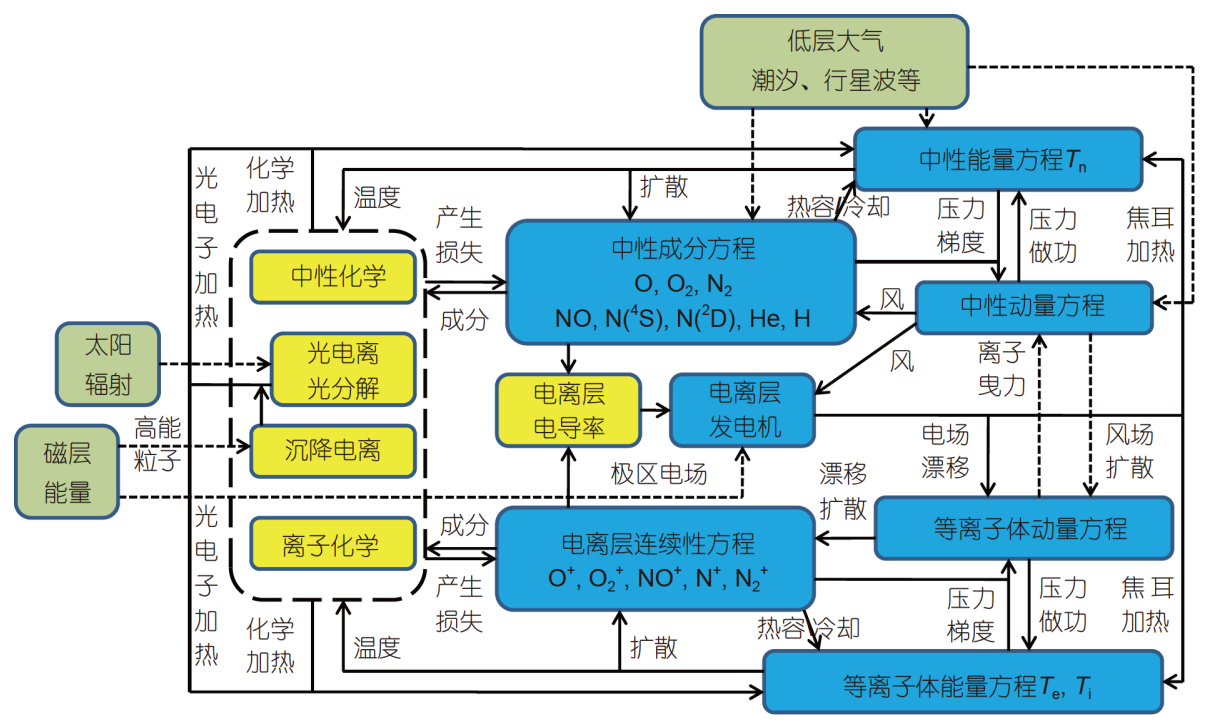

图 2 GCITEM-IGGCAS模式框架

Figure 2 The structure of GCITEM-IGGCAS model 

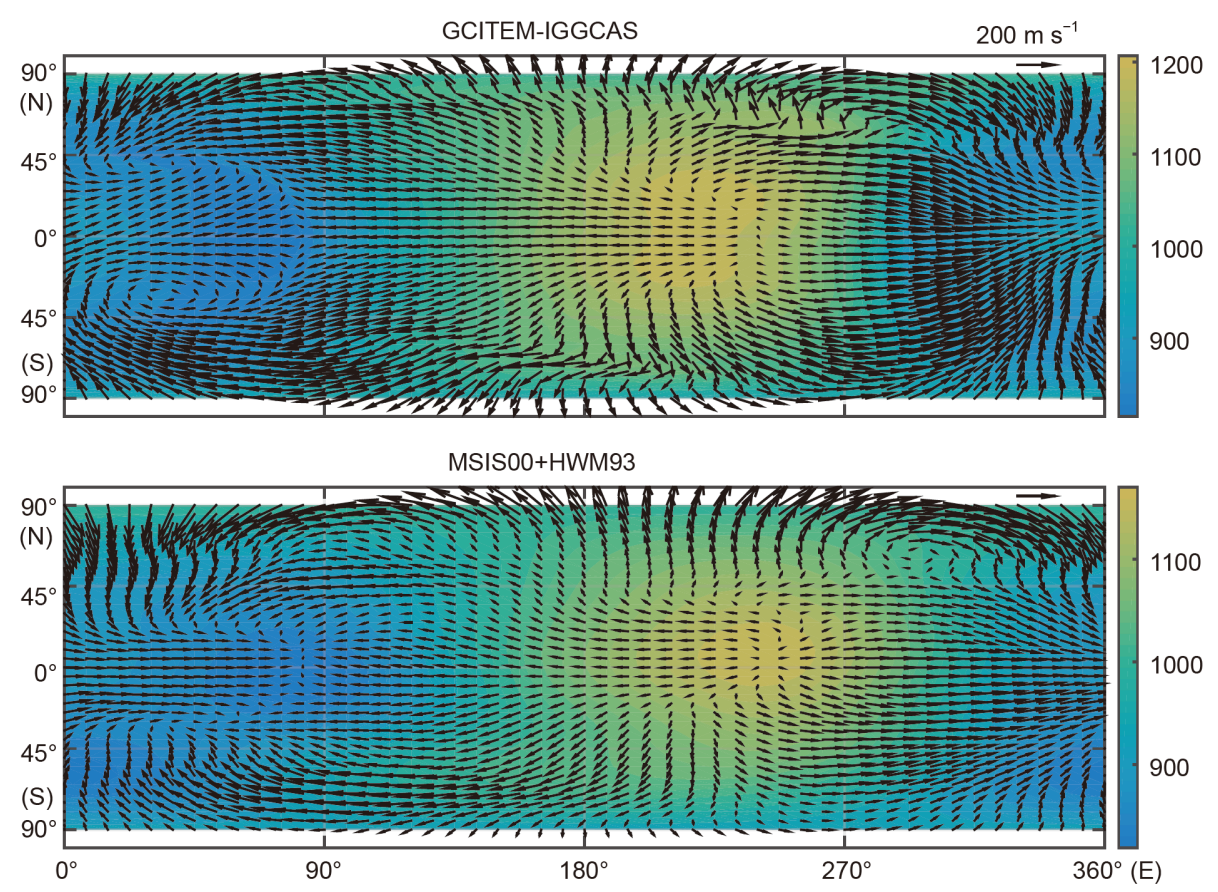

图 3 中等太阳活动下春分世界时 0 时 $405 \mathrm{~km}$ 处中性温度和中性风场随经度(地方时)和纬度的分布. 箭头表示中性风的强度和方向. (a) GCITEM-IGGCAS模式的模拟结果; (b) MSIS00和HWM93经验模型的结果

Figure 3 Neutral temperature (K) and horizontal neutral wind velocity (m/s) from GCITEM-IGGCAS simulation (a) and from MSIS00/HWM93 empirical model (b) at the height of $405 \mathrm{~km}$ at 0000 UT for March Equinox

表 1 目前主流的地球高层大气理论模式

Table 1 Current Earth's upper atmospheric theoretical models

\begin{tabular}{ccccccc}
\hline 模式名称 & 坐标系 & 全球覆盖 & 包含发电机过程 & 地磁场 & 真实大气模拟能力 & 开发国家 \\
\hline TIEGCM & 地理-压力 & 是 & 是 & 非偶极场 & 较强 & 美国 \\
CTIPE & 地理-压力 & 是 & 是 & 偶极场 & 较强 & 英国-美国 \\
GSM TIP & 地磁-高度 & 是 & 仅E区发电机 & 偶极场 & 较强 & 伐罗斯 \\
GCITEM-IGGCAS & 地理-高度 & 是 & 是 & 非偶极场 & 较强 & 中国 \\
GITM & 地理-高度 & 是 & 否 & 非偶极场 & 较强 & 美国 \\
\hline
\end{tabular}

对比表. 由表1可见，目前国内外现有的高层大气理论 模式普遍具有下列特点: (1) 现有高层大气理论模式全 部为全球三维模式, 且部分能够使用真实的非偶极地 磁场, 能够系统模拟电离层/热层参数的高度、纬度和 经度变化, 并能够有效再现真实的地球高层大气时空 变化特征，有利于同观测数据之间的对比研究以及高 层大气区域特性的研究; (2) 现有高层大气理论模式普 遍是一个全自洽、全耦合的电离层/热层模式. 能够自 洽求解几乎全部的电离层/热层参数(包括中性大气、 电子和离子的温度、密度、成分、速度、电场、产生/ 损失率和加热/冷却率等), 并在每个时间步和每个空间
格点都实现了这些参数之间的耦合; (3) 模式适应性强, 可以很方便地选择不同的上下边界条件、不同的地磁 场模型和不同的极区能量注人(高纬电离层电场和极 区高能粒子沉降), 有利于具体物理问题的研究; (4) 随 着计算条件的进步, 目前的高层大气模式普遍在普通 的PC机就可以运行, 极大地降低了科研成本提高了工 作效率. 国内外现有的高层大气理论模式基本上已经 可以模拟出不同地球物理条件下的高层大气系统中等 离子体和中性成分的温度、密度、风场和成分等参数 的主要气候学分布特征, 并与已有的电离层/热层探测 结果以及经验模型基本一致. 
近年来，对于高层大气理论模式的发展主要以对 现有模式的改进为主, 逐步提高模拟能力. 具体包括提 高外界能量和动量输人的精度, 主要包括发展更精细 的太阳辐射模型、给出更为精确的高纬能量和动量注 人、低热层大气波动注人等 ${ }^{[44]}$; 进一步修正模式内部 物理参数和过程参数化方案, 引人一些新的物理化学 机制 ${ }^{[45,46]}$; 尝试发展高分辨率的高层大气模式, 模拟中 小尺度的高层大气物理过程 ${ }^{[47]}$; 发展观测数据同化技 术, 为开发空间天气实时预报模式准备基础 ${ }^{[48]}$.

\section{3 行星高层大气建模}

高层大气理论模式在高层大气(包括电离层)研究 中起到了重要作用, 不仅帮助我们进一步认识了高层 大气的时空变化和物理过程，而且在缺少观测的情况 下在高层大气参数估计和参数反演中起到了重要作用. 后一点不仅在地球的高层大气(包括电离层)研究中起 到了重要作用, 更在其他行星的高层大气研究中发挥 了不可替代的作用.

自从 20 世纪 60 年代人类开始星际航行以来，人类 对于太阳系其他行星的高层大气(含电离层)进行了大 量观测, 取得了丰硕的研究成果. 然而, 总体而言我们 对于其他行星高层大气的观测仍然处于相对稀缺状态, 对其他行星高层大气的认识仍然有很多缺陷，其他行 星高层大气理论建模也更有挑战性，相应理论模式也 需要使用更多的参数化过程. 因而, 与开发地球模式时 类似，学界开发了一系列能够模拟全球平均场并测试 相应参数化过程的行星的一维高层大气理论模式. 总 体而言, 这些高层大气一维模型可以分为两类: 不考虑 垂直输运过程的主要基于光化学平衡假设的高层大气 模型、考虑了不同成分垂直输运过程的高层大气模型.
光化学平衡模型没有考虑不同高度之间的耦合，只考 虑了各种化学过程, 在早期的行星高层大气和电离层 研究中应用比较多, 主要与其他行星的高层大气掩星 观测数据相配合，在缺乏探测的情况下分析行星高层 大气密度和化学成分的高度变化 ${ }^{[49 \sim 53]}$. 由于缺少化学 成分的输运过程, 光化学平衡模型不能模拟绝大部分 中性化学成分和长寿命离子的时间变化规律，在使用 上受到了很多限制. 因此, 光化学平衡模型逐步被考虑 了不同成分垂直输运过程的高层大气模型所代 替 ${ }^{[54-61]}$. 与Roble等人 ${ }^{[11]}$ 开发 TIGCM模式前先行开发一 个测试用的一维模式类似，近年来开发的其他星球一 维高层大气模型都尽可能包括了与相应的更高维模型 类似的物理化学过程，在更高维模型的开发中扮演了 探路者的角色，并在后续的行星高层大气物理研究和 更高维行星大气模型改进中一直起着重要作用 ${ }^{[57 \sim 61]}$.

由于对其他行星高层大气的认识相对贯乏，对于 其他行星更高维高层大气理论建模通常需要借鉴地球 高层大气理论建模的成果，如地球相应模型的结构框 架、基本的物理假设等. 因此, 虽然由于大气化学成分 等差异, 导致其他行星高层大气理论模式与地球模式 间有很大差异，但是目前国际上比较认可的行星高层 大气理论模式大部分是以目前主流的地球高层大气理 论模式为开发基础，某种意义上可以认为是这些模型 在其他行星的衍生版本. 表 2 总结了国际上比较认可的 行星高层大气理论模式，可见其中的大部分都有相应 的地球高层大气模型作为开发基础. 由于以地球的模 式作为开发基础, 起点较高, 所以行星高层大气理论模 式的开发大都直接跳过了地球高层大气理论模式的二 维开发阶段, 直接开发三维模式. 下面以行星为单位对 表2中的行星高层大气理论模型进行简单介绍.

表 2 目前主流的行星高层大气理论模式

Table 2 Current planet's upper atmospheric theoretical models

\begin{tabular}{ccccc}
\hline 模式名称 & 对应星球 & 是否有一维模式 & 对应的地球模式 & 开发国家 \\
\hline VTGCM & 金星 & 是 & TIEGCM & 美国 \\
MTGCM & 火星 & 是 & TIEGCM & 美国 \\
MGITM & 火星 & 是 & GITM & 美国 \\
JTGCM & 木星 & TIEGCM & 美国 \\
JIM & 木星 & 否 & CTIM & 英国 \\
STIM & 土星 & 否 & 无 & 美国-英国 \\
TTIM & 土卫六 & 否 & 无 & 美国-英国 \\
TGITM & 土卫六 & GITM & 美国 \\
\hline
\end{tabular}


太阳系的行星一般分为三类：类地行星、巨行星 和远日行星. 其中的类地行星是与地球相类似的行星, 距离太阳近, 体积和质量都较小, 平均密度较大, 主要 包括水星、地球、火星、金星. 类地行星中水星距离 太阳过近, 基本没有大气层, 不需要高层大气模式. 金 星和火星有较厚的大气层, 但是其大气的主要成分以 $\mathrm{CO}_{2}$ 为主，与地球大气有明显差异. 目前，对于类地行 星的高层大气建模以金星和火星为主.

目前, 国际上比较知名的金星高层大气理论模式 是美国的VTGCM模式(venus thermosphere general circulation model). VTGCM模式是以前文提到的美国 NCAR的TGCM模式为基础开发的一套金星高层大气 三维有限差分流体动力学模型. 自该模型于1988年建 立以来, 经历了不断的改进 ${ }^{[62 \sim 68]}$. 该模式采用压力坐标 系, 模式的纬度-经度分辨率为 $5^{\circ} \times 5^{\circ}$, 在垂直方向上把 80 200 km的金星高层大气均匀分为46个等压面. 该模 式能够模拟金星高层大气的 4 种主要中性化学成分 $\left(\mathrm{CO}_{2} 、 \mathrm{CO} 、 \mathrm{O}\right.$ 和 $\left.\mathrm{N}_{2}\right)$ 的数密度、4种次要中性化学成分 $\left(\mathrm{O}_{2} 、 \mathrm{NO} 、 \mathrm{~N}\left({ }^{4} \mathrm{~S}\right)\right.$ 和 $\left.\mathrm{N}\left({ }^{2} \mathrm{D}\right)\right)$ 的数密度、日侧主要离子成 分 $\left(\mathrm{CO}_{2}^{+} 、 \mathrm{O}_{2}^{+} 、 \mathrm{O}^{+}\right.$和 $\left.\mathrm{NO}^{+}\right)$的数密度、中性温度和中性 风的三维时空分布. 目前该模式已经包含了相对较为 完备的化学反应框架，能够较为合理地模拟金星高层 大气化学成分的演化特征. 该模式对于电离层的模拟 主要基于光化学平衡假设, 没有考虑等离子体的输运 过程, 对于长寿命离子 $\left(\right.$ 如 $\left.\mathrm{O}^{+}\right)$的模拟能力有限, 且电子 和离子温度由经验模型提供, 没能实现自洽计算. 该模 式对于金星高层大气的加热过程主要考虑了半自洽的 太阳极紫外、远紫外和红外辐射加热过程, 对于冷却 过程主要考虑 $\mathrm{CO}_{2} \quad 15 \mu \mathrm{m}$ 的非局地热平衡(NLTE)红外 辐射冷却过程. VTGCM模式还能够模拟计算金星高层 大气 $\mathrm{O}_{2}$ 可见光气辉(波长400 800 nm)、 $\mathrm{O}_{2}$ 红外气辉(波 长1.27 $\mu \mathrm{m}$ )和 $\mathrm{NO}$ 紫外夜气辉(波长 $198.0 \mathrm{~nm}$ )的时空分 布, 并广泛应用于相关研究中.

火星与金星高层大气虽然都是以 $\mathrm{CO}_{2}$ 为主, 但是由 于两者重力、自转、星日距离等的差别, 两者的高层 大气有显著的不同. 因此, 国际上也独立开发了一系列 火星高层大气理论模式. 目前, 国际上比较知名的火星 高层大气理论模式之一是美国的MTGCM模式(Mars thermosphere general circulation model). 与VTGCM模式 类似，MTGCM模式也是以前文提到的美国NCAR的 TGCM模式为基础开发的一套火星高层大气三维有限 差分流体动力学模型. 自该模型于1999年建立以来, 经
历了不断的改进 ${ }^{[6773]}$. 与其他基于 TGCM的模式类似， 该模式也采用了压力坐标系, 模式的纬度-经度分辨率 为 $5^{\circ} \times 5^{\circ}$, 在垂直方向上把 70 300 km的火星高层大气 均匀分为 33 个等压面. 该模式能够模拟金星高层大气 的4种主要中性化学成分 $\left(\mathrm{CO}_{2} 、 \mathrm{CO} 、 \mathrm{O}\right.$ 和 $\left.\mathrm{N}_{2}\right)$ 的数密 度、4种次要中性化学成分 $\left(\mathrm{Ar} 、 \mathrm{He} 、 \mathrm{O}_{2} 、 \mathrm{NO}\right.$ 和 $\left.\mathrm{N}\left({ }^{4} \mathrm{~S}\right)\right)$ 的数密度、日侧主要离子 $\left(\mathrm{CO}_{2}^{+} 、 \mathrm{CO}^{+} 、 \mathrm{O}_{2}^{+} 、 \mathrm{O}^{+}\right.$、 $\mathrm{N}_{2}^{+}$和 $\mathrm{NO}^{+}$)的数密度、中性温度和中性风的三维时空 分布. 与VTGCM类似, 目前该模式也已经包含了相对 较为完备的化学反应框架, 能够较为合理地模拟火星 高层大气化学成分的演化特征. 该模式对于电离层的 模拟主要基于光化学平衡假设, 没有考虑等离子体的 输运过程, 对于长寿命离子 $\left(\right.$ 如 $\left.\mathrm{O}^{+}\right)$的模拟能力有限, 且 电子和离子温度主要由经验模型提供, 没能实现自洽 计算. 该模式对于火星高层大气的加热过程主要考虑 了半自洽的太阳极紫外、远紫外和红外辐射加热过程, 对于冷却过程主要考虑 $\mathrm{CO}_{2} \quad 15 \mu \mathrm{m}$ 的红外辐射冷却过 程. 但是与VTGCM不同，MTGCM中引人了一种可实 现快速计算的火星 $\mathrm{CO}_{2} 15 \mu \mathrm{m}$ 的NLTE红外辐射冷却计 算方案 ${ }^{[72]}$, 该方案是基于相关的火星大气一维NLTE模 型的详细计算结果 ${ }^{[74]}$. 此外, 该模式通过与美国开发的 火星的中低层大气理论模式MGCM(Mars general circulation model)相耦合, 形成火星的全大气理论模式 MGCM-MTGCM(Mars general circulation model-Mars thermosphere general circulation model), 实现了对火星 的全大气模拟, 能够研究火星热层对于火星各种大气 潮汐的响应.

另一种国际上比较知名的火星高层大气理论模式 是美国的MGITM模式(Mars global ionosphere thermosphere model ${ }^{[75]}$ ). 该模式以前文提到的美国密歇根大学 的GITM模式为基础, 通过引人火星的基本参数、各种 常数和关键的辐射过程而开发的一个火星全大气三维 流体动力学模型. 该模式采用了高度坐标系, 模式的纬 度-经度分辨率为 $5^{\circ} \times 5^{\circ}$, 在垂直方向上把 $0 \sim 250 \mathrm{~km}$ 的火 星高层大气均匀分为约 100 个等高面. 该模式能够模拟 金星高层大气的6种主要中性化学成分 $\left(\mathrm{CO}_{2} 、 \mathrm{CO} 、\right.$ $\mathrm{O}_{2} 、 \mathrm{O} 、 \mathrm{~N}_{2}$ 和 $\left.\mathrm{Ar}\right)$ 的数密度、日侧主要离子 $\left(\mathrm{CO}_{2}{ }^{+}\right.$、 $\mathrm{O}_{2}^{+} 、 \mathrm{O}^{+} 、 \mathrm{~N}_{2}^{+}$和 $\left.\mathrm{NO}^{+}\right)$的数密度、中性温度和中性风的 三维时空分布, 并计划在改进版中实现对 $\mathrm{N}\left({ }^{4} \mathrm{~S}\right) 、 \mathrm{~N}$ $\left({ }^{2} \mathrm{D}\right) 、 \mathrm{NO} 、 \mathrm{He} 、 \mathrm{H}_{2}$ 和 $\mathrm{H}$ 等次要中性化学成分的模拟。 与MTGCM类似, 该模式也包含了相对较为完备的化学 反应框架, 并主要基于光化学平衡假设模拟电离层. 与 
地球的GCITEM模式类似，MGITM也放弃了垂直方向 上的静力平衡假设, 扩展了模式的适用范围.

随着国内火星探测计划的推进，也开始研发火星 的高层大气理论模式. 任志鹏等人 ${ }^{[76]}$ 提出以国内的地 球电离层热层耦合模式GCITEM-IGGCAS为基础, 开 发相应的火星电离层热层耦合模式. 该模式计划采用 高度坐标系，纬度-经度分辨率为 $5^{\circ} \times 7.5^{\circ}$, 在垂直方向 上把70 300 $\mathrm{km}$ 的火星高层大气均匀分为 35 个等压面, 模拟火星高层大气的4种主要中性化学成分 $\left(\mathrm{CO}_{2}\right.$ 、 $\mathrm{CO} 、 \mathrm{O}$ 和 $\left.\mathrm{N}_{2}\right)$ 的数密度、4种次要中性化学成分 $(\mathrm{Ar}$ 、 $\mathrm{He} 、 \mathrm{O}_{2} 、 \mathrm{NO} 、 \mathrm{~N}\left({ }^{4} \mathrm{~S}\right)$ 和 $\left.\mathrm{N}\left({ }^{2} \mathrm{D}\right)\right)$ 的数密度、日侧主要离 子 $\left(\mathrm{CO}_{2}^{+} 、 \mathrm{CO}^{+} 、 \mathrm{O}_{2}^{+} 、 \mathrm{O}^{+} 、 \mathrm{~N}_{2}^{+}\right.$和 $\left.\mathrm{NO}^{+}\right)$的数密度、中性 温度和中性风的三维时空分布.

除了类地行星外，巨行星也是目前研究比较多的 行星. 巨行星又称类木行星, 包括木星和土星, 是不以 岩石或其他固体为主要成分构成的大行星. 他们体积 巨大，质量也大，但密度小，主要由氢、氦、氛等轻元 素组成. 类地行星的高层大气也以氢、氦为主要成分, 表现出与类地行星迥异的性质, 因此国际上也为木星 和土星开发了相应的高层大气理论模式.

目前，国际上比较知名的木星高层大气理论模式 之一是英国的JIM(Jupiter ionosphere model)模式. 它由 Achilleos等人 ${ }^{[77]}$ 在英国以地球的CTIM电离层/热层耦 合模式为基础开发，是国际上第一个木星高层大气理 论模式. 该模式成功模拟了 $2 \mu \mathrm{bar}$ 等压面以上的木星电 离层和热层主要参数的时空变化, 包括木星高层大气 的3种主要中性化学成分 $\left(\mathrm{H} 、 \mathrm{H}_{2}\right.$ 和 $\left.\mathrm{He}\right)$ 的数密度、 3 种 主要离子 $\left(\mathrm{H}_{3}{ }^{+} 、 \mathrm{H}_{2}{ }^{+}\right.$和 $\left.\mathrm{H}^{+}\right)$的数密度、中性温度和中性 风. 该模式自洽地计算了太阳辐射加热和光电离过程, 在动量方程求解过程中考虑了扩散过程以及离子间中 性碰撞的影响, 在中性成分计算中考虑了风的输运过 程和扩散过程的影响，在模式内还包括了高纬地区的 高能粒子沉降过程和焦热加热过程.

美国的JTGCM模式(Jupiter thermosphere general circulation model)则是另一个比较著名的木星高层大 气理论模式. 与VTGCM和MTGCM模式类似, JTGCM 模式也是以地球的 TGCM模式为基础开发的一套木星 高层大气三维有限差分流体动力学模型. 该模型于 2005 年建立 ${ }^{[78-80]}$, 采用了压力坐标系, 模式的纬度-经 度分辨率为 $5^{\circ} \times 5^{\circ}$, 在垂直方向上把 $20 \mu \mathrm{bar} \sim 1 \times 10^{-4}$ nbar的木星高层大气均匀分为 39 个等压面. 该模式能 够模拟木星高层大气的 3 种主要中性化学成分 $\left(\mathrm{H} 、 \mathrm{H}_{2}\right.$
和 $\mathrm{He})$ 的数密度、2 种次要中性化学成分 $\left(\mathrm{C}_{2} \mathrm{H}_{2}\right.$ 和 $\left.\mathrm{CH}_{4}\right)$ 的数密度、 3 种主要离子 $\left(\mathrm{H}_{3}{ }^{+} 、 \mathrm{H}_{2}{ }^{+}\right.$和 $\left.\mathrm{H}^{+}\right)$的数密度、中 性温度和中性风的三维时空分布. 不同于主要由太阳 辐射加热控制的类地行星高层大气环流，木星高层大 气环流主要由极光和焦耳加热过程来驱动, 因而 TGCM模式表现出迥异于类地行星模型的一系列特征. 学界应用这两个模式在木星热层能量收支等方面取得 了一系列突破性的成果 ${ }^{[79-81]}$.

除了木星模式以外，学界也开发了相应的土星高 层大气理论模式. Müller-Wodarg等人 ${ }^{[82]}$ 开发了世界上 第一个土星热层和电离层的高层大气环流模式, 并命 名为STIM模式(Saturn thermosphere ionosphere model). 该模式是由美国波士顿大学、英国的伦敦帝国理工学 院和伦敦大学学院联合开发. 它在压力坐标系下求解 了100 nbar等压面以上土星高层大气非线性的能量、 动量和连续性方程. 学界应用该模式在土星热层能量 收支等方面取得了一系列突破性成果 ${ }^{[82]}$.

除了这些行星大气高层模式外，国际上也对太阳 系内一些特殊卫星的高层大气展开了研究. 如, 土卫六 (Titan, 又称为泰坦星)是环绕土星运行的一颗卫星, 是 土星卫星中最大的一个, 也是太阳系第二大卫星, 是目 前太阳系内唯一拥有牢固的永久大气层的卫星. Müller-Wodarg等人 ${ }^{[83]}$ 开发了世界上第一个土卫六热层和 电离层的高层大气环流模式, 并命名为 TTIM模式(Ti$\tan$ thermosphere ionosphere model). 该模式由美国波 土顿大学、亚利桑那大学和英国的伦敦大学学院联合 开发. 最初, 该模式以地球的UCL-GCM模式 ${ }^{[3]}$ 为基础 进行开发, 但是最后改为全新独立开发. 此外, 美国也 以地球的GTIM模式为基础开发了 TGTIM土卫六高层 大气理论模式 ${ }^{[57,61,84]}$.

虽然经过学界几十年来的努力, 国际上在行星高 层大气理论建模方面取得了长足的进步，并在行星高 层大气研究以及相关的行星空间环境和行星大气研究 中做出了巨大的贡献 ${ }^{[85]}$. 然而, 由于目前对于行星高层 大气的认识仍然普遍不足, 相对于地球高层大气理论 模式而言, 行星高层大气理论模式普遍存在包含的物 理过程相对简单、对真实行星大气再现能力差、模拟 过程自洽性不足依赖经验模型或观测数据输人的情况. 图4给出了我国计划开发的火星高层大气理论模式的 框架，相对于图2给出的作为该模式基础的GCITEM模 式的框架明显更为简单, 可见其在模式内部自洽性、 包含物理过程的完备性等方面有着显著的不足，因而 


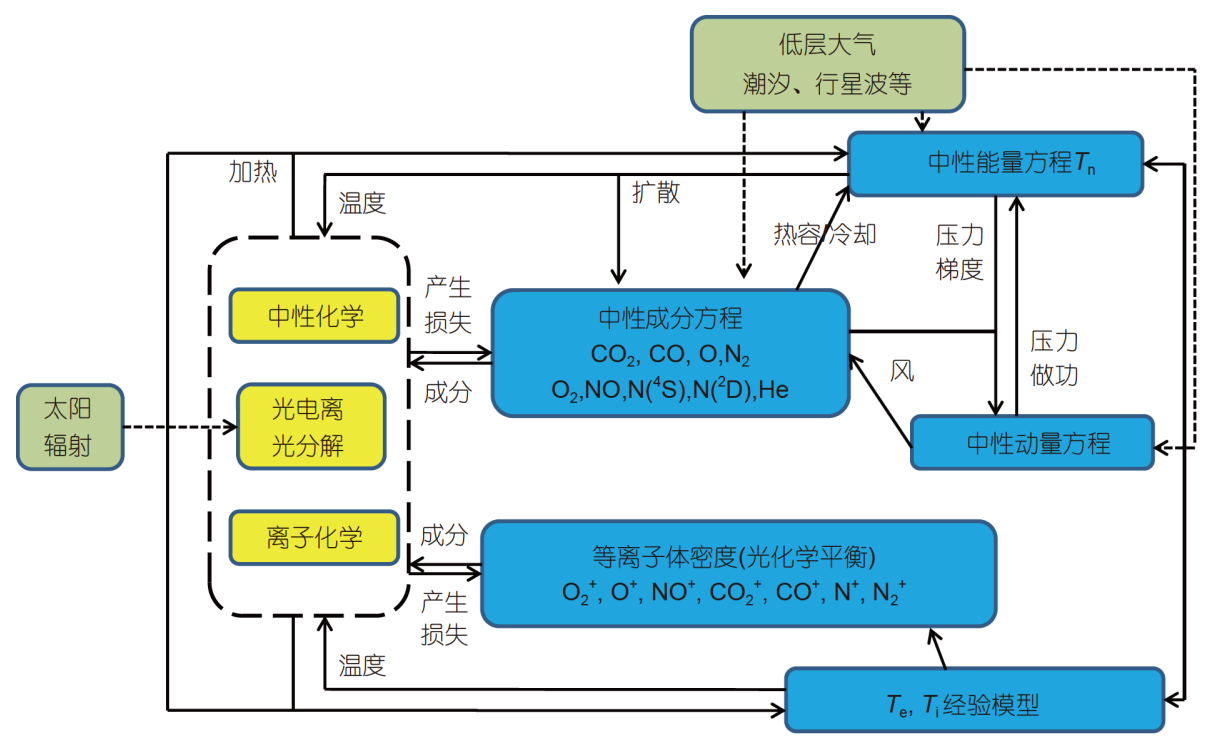

图 4 火星高层大气理论模式框架

Figure 4 The structure Mars' upper atmospheric theoretical models

行星高层大气理论建模研究仍然有很长的路要走, 是 值得国内科研界大力推进的行星科学研究方向.

高层大气作为大气层与空间环境的过渡区, 其建 模兼有大气理论建模与空间环境理论建模特点, 因而 开展行星高层大气理论建模研究首先需要培养一支专 门的高水平的行星高层大气理论建模研究队伍. 由表 2 可见目前主流的行星高层大气理论模式主要都是以地 球高层大气理论模式为基础开发而来, 因此首先锻炼 一支高水平的地球高层大气理论建模研究队伍, 进而 由该团队进一步开展行星高层大气理论建模研究就是 一条可行的团队建设路线. “鸿鹄专项”对于地球临近 空间-高层大气耦合理论模式开发, 就是正在构建一支 高层大气理论建模研究团队, 进而可以有效地支撑国 内后续的行星高层大气理论建模研究.

\section{4 小结}

地球和行星的高层大气作为大气圈与空间环境的
过渡区, 是大气科学和空间科学的交叉研究领域. 高层 大气理论模式及其数值模拟可以突破实验观测的限制, 又能够比理论分析更为全面细致地重现高层大气的各 种物理和化学过程, 在高层大气的研究中具有独特的 优势, 一直起着重要作用. 虽然地球和其他行星高层大 气存在差异, 但地球和其他行星高层大气理论模式有 着密切的联系, 大多数的行星高层大气理论模式都是 以地球高层大气理论模式为基础开发的. 本文聚焦于 地球和行星高层大气理论模式，对目前国内外主流的 地球和行星高层大气理论模式的发展历史及其性能进 行了系统介绍. 总体而言, 经过近半个世纪的努力, 地 球和行星高层大气理论模式取得了长足的进步. 相对 而言, 由于观测和基础研究更为充分, 地球的高层大气 理论模式发展的较为完备, 目前已经具备了向高层大 气数值预报方向发展的潜力. 由于观测与基础研究的 不足, 行星高层大气理论模式发展的相对滞后, 是值得 大力推进的行星科学研究方向.

\section{参考文献}

1 Kohl H, King J W. Atmospheric winds between 100 and $700 \mathrm{~km}$ and their effects on the ionosphere. J Atmos Terrestrial Phys, 1967, 29: 1045-1062

2 Lindzent R S, Hong S S. Equivalent gravity modes-An interim evaluation. Geophys Fluid Dyn, 1973, 4: 279-292

3 Fuller-Rowell T J, Rees D. A three-dimensional time-dependent global model of the thermosphere. J Atmos Sci, 1980, 37: 2545-2567

4 Dickinson R E, Ridley E C, Roble R G. A three-dimensional general circulation model of the thermosphere. J Geophys Res, 1981, 86: 1499-1512

5 Dickinson R E, Ridley E C, Roble R G. Meridional circulation in the thermosphere I. Equinox conditions. J Atmos Sci, 1975, 32: 1737-1754

6 Dickinson R E, Ridley E C, Roble R G. Meridional circulation in the thermosphere. II. Solstice conditions. J Atmos Sci, 1977, 34: 178-192

7 Dickinson R E, Ridley E C. Numerical solution for the composition of a thermosphere in the presence of a steady subsolar to-antisolar circulation 
with application to venus. J Atmos Sci, 1972, 29: 1557-1570

8 Dickinson R E, Ridley E C, Roble R G. Thermospheric general circulation with coupled dynamics and composition. J Atmos Sci, 1984, 41: 205219

9 Roble R G, Dickinson R E, Ridley E C. Global circulation and temperature structure of thermosphere with high-latitude plasma convection. J Geophys Res, 1982, 87: 1599-1614

10 Roble R G, Ridley E C. An auroral model for the NCAR thermospheric general circulation model (TGCM). Ann Geophys, 1987, 5: 369-382

11 Roble R G, Ridley E C, Dickinson R E. On the global mean structure of the thermosphere. J Geophys Res, 1987, 92: 8745-8758

12 Roble R G, Ridley E C, Richmond A D, et al. A coupled thermosphere/ionosphere general circulation model. Geophys Res Lett, 1988, 15: 13251328

13 Forbes J M, Roble R G, Fesen C G. Acceleration, heating, and compositional mixing of the thermosphere due to upward propagating tides. J Geophys Res, 1993, 98: 311-321

14 Richmond A D, Ridley E C, Roble R G. A thermosphere/ionosphere general circulation model with coupled electrodynamics. Geophys Res Lett, 1992, 19: 601-604

15 Richmond A D. Ionospheric electrodynamics using magnetic apex coordinates. J Geomagn Geoelec, 1995, 47: 191-212

16 Roble R G. Energetics of the mesosphere and thermosphere. In: Johnson R M, Killeen T L, eds. The Upper Mesosphere and Lower Thermosphere: A Review of Experiment and Theory. Geophys Monogr Ser, Vol. 87. Washington DC: American Geophysics Union, 1995

17 Roble R G, Ridley E C. A thermosphere-ionosphere-mesosphere-electrodynamics general circulation model (time-GCM): Equinox solar cycle minimum simulations (30-500 km). Geophys Res Lett, 1994, 21: 417-420

18 Wang W. A thermosphere-ionosphere nested grid model. Doctor Dissertation. Michigan: The University of Michigan, 1997

19 Wang W, Killeen T L, Burns A G, et al. A high-resolution, three-dimensional, time dependent, nested grid model of the coupled thermosphereionosphere. J Atmos Sol-Terr Phys, 1999, 61: 385-397

20 Peymirat C, Richmond A D, Emery B A, et al. A magnetosphere-thermosphere-ionosphere electrodynamics general circulation model. J Geophys Res, 1998, 103: 17467-17477

21 Wang W, Wiltberger M, Burns A G, et al. Initial results from the coupled magnetosphere-ionosphere-thermosphere model: Thermosphereionosphere responses. J Atmos Sol-Terr Phys, 2004, 66: 1425-1441

22 Mendillo M, Rishbeth H, Roble R G, et al. Modelling F2-layer seasonal trends and day-to-day variability driven by coupling with the lower atmosphere. J Atmos Sol-Terr Phys, 2002, 64: 1911-1931

23 Rees D, Fuller-Rowell T J, Smith R W. Measurements of high latitude thermospheric winds by rocket and ground-based techniques and their interpretation using a three-dimensional, time-dependent dynamical model. Planet Space Sci, 1980, 28: 919-932

24 Fuller-Rowell T J, Rees D. Derivation of a conservation equation for mean molecular weight for a two-constituent gas within a three-dimensional, time-dependent model of the thermosphere. Planet Space Sci, 1983, 31: 1209-1222

25 Quegan S, Bailey G J, Moffett R J, et al. A theoretical study of the distribution of ionization in the high-latitude ionosphere and the plasmasphere: First results on the mid-latitude trough and the light-ion trough. J Atmos Terr Phys, 1982, 44: 619-640

26 Millward G H. A global model of the earth's thermosphere, ionosphere and plasmasphere: Theoretical studies of the response to enhanced highlatitude convection. Doctor Dissertation. Sheffield: University of Sheffield, 1993

27 Fuller-Rowell T J, Rees D, Quegan S, et al. Interactions between neutral thermospheric composition and the polar ionosphere using a coupled ionosphere-thermosphere model. J Geophys Res, 1987, 92: 7744-7748

28 Millward G H, Moffett R J, Quegan W, et al. A coupled thermospheric-ionospheric-plasmasphere Model (CTIP). In: Schunk R W, ed. STEP: Handbook of Ionospheric Models. Utath: Utath State University, 1996. 173

29 Millward G H, Müller-Wodarg I C F, Aylward A D, et al. An investigation into the influence of tidal forcing on $F$ region equatorial vertical ion drift using a global ionosphere-thermosphere model with coupled electrodynamics. J Geophys Res, 2001, 106: 24733-24744

30 Harris M J, Arnold N F, Aylward A D. A study into the effect of the diurnal tide on the structure of the background mesosphere and thermosphere using the new coupled middle atmosphere and thermosphere (CMAT) general circulation model. Ann Geophys, 2002, 20: 225-235

31 Namgaladze A A, Korenkov Y N, Klimenko V V, et al. Global model of the thermosphere-ionosphere-protonosphere system. Pure Appl Geophys, 1988, 127: 219-254

32 Namgaladze A A, Korenkov Y N, Klimenko V V, et al. A global numerical model of the thermosphere, ionosphere, and protonosphere of the earth. Geomag Aeron, 1990, 30: 515-521

33 Namgaladze A A, Korenkov Y N, Klimenko V V, et al. Numerical modelling of the thermosphere-ionosphere-protonosphere system. J Atmos Terr Phys, 1991, 53: 1113-1124

34 Ridley A J, Deng Y, Tóth G. The global ionosphere-thermosphere model. J Atmos Sol-Terr Phys, 2006, 68: 839-864

35 Miyoshi Y, Fujiwara H. Day-to-day variations of migrating diurnal tide simulated by a GCM from the ground surface to the exobase. Geophys Res 
Lett, 2003, 30: 1789

36 Akmaev R A, Fuller-Rowell T J, Wu F, et al. Tidal variability in the lower thermosphere: Comparison of Whole Atmosphere Model (WAM) simulations with observations from TIMED. Geophys Res Lett, 2008, 35: 10-29

37 Fuller-Rowell T J, Akmaev R A, Wu F, et al. Impact of terrestrial weather on the upper atmosphere. Geophys Res Lett, 2008, 35: L09808

38 Liu H L, Bardeen C G, Foster B T, et al. Development and validation of the Whole Atmosphere Community Climate Model with thermosphere and ionosphere extension (WACCM-X). J Adv Model Earth Sys, 2018, 10: 381-402

39 Liu H L, Foster B T, Hagan M E, et al. Thermosphere extension of the Whole Atmosphere Community Climate Model. J Geophys Res, 2010, 115: A12302

40 Wang J S, Xiao Z. Thermospheric circulation model in meridian plane I-Storm time variations in thermal status and circulation. Sci China Ser E Tech Sci, 2000, 43: 577-585

41 Lei J, Liu L, Luan X, et al. Model study on neutral winds in ionospheric F-region and comparison with the equivalent winds derived from the Wuhan ionosonde data. Terr Atmos Oceanic Sci, 2003, 14: 1-12

42 Ren Z, Wan W, Liu L. GCITEM-IGGCAS: A new global coupled ionosphere-thermosphere-electrodynamics model. J Atmos Sol-Terr Phys, 2009, 71: 2064-2076

43 Ren Z, Wan W, Wei Y, et al. A theoretical model for mid- and low-latitude ionospheric electric fields in realistic geomagnetic fields. Chin Sci Bull, 2008, 53: 3883-3890

44 Richmond A D, Kamide Y. Mapping electrodynamic features of the high-latitude ionosphere from localized observations: Technique. J Geophys Res, 1988, 93: 5741-5759

45 Maruyama N. Dynamic and energetic coupling in the equatorial ionosphere and thermosphere. J Geophys Res, 2003, $108: 1396$

46 Lei J, Thayer J P, Wang W, et al. Simulations of the equatorial thermosphere anomaly: Field-aligned ion drag effect. J Geophys Res, 2012, 117: A01304

47 Dang T, Lei J, Wang W, et al. Suppression of the polar tongue ofionization during the 21 August 2017solar eclipse. Geophys Res Lett, 2018, 45: 2918-2925

48 Yue X, Wang W, Lei J, et al. Long-lasting negative ionospheric storm effects in low and middle latitudes during the recovery phase of the 17 March 2013 geomagnetic storm. J Geophys Res Space Phys, 2016, 121: 9234-9249

49 McElroy M B. The upper atmosphere of Mars. Astrophys J, 1967, 150: 1125-1138

50 McElroy M B. The upper atmosphere of Venus. J Geophys Res, 1968, 73: 1513-1521

51 McElroy M B. Structure of the Venus and Mars atmospheres. J Geophys Res, 1969, 74: 29-41

52 Stewart R W. Interpretation of Mariner 5 and Venera 4 data on the upper atmosphere of Venus. J Atmos Sci, 1968, 25: 578-579

53 Stewart R W. The electron distributions in the Mars and Venus upper atmospheres. J Atmos Sci, 1971, 28: 1069-1073

54 Fox J L. Response of the Martian thermosphere/ionosphere to enhanced fluxes of solar soft X rays. J Geophys Res, 2004, 109: A11310

55 Fox J L, Sung K Y. Solar activity variations of the Venus thermosphere/ionosphere. J Geophys Res, 2001, 106: 21305-21335

56 Fox J L, Yeager K E. Morphology of the near-terminator Martian ionosphere: A comparison of models and data. J Geophys Res, 2006, 111: A10309

57 Bell J M. The dynamics of the upper atmospheres of Mars and Titan. Doctor Dissertation. Michigan: University of Michigan, 2008

58 Bell J M, Bougher S W, Waite Jr J H, et al. Simulating the one-dimensional structure of Titan's upper atmosphere: 1. Formulation of the Titan Global Ionosphere-Thermosphere Model and benchmark simulations. J Geophys Res, 2010, 115: E12002

59 Bell J M, Bougher S W, Waite Jr J H, et al. Simulating the one-dimensional structure of Titan's upper atmosphere: 2. Alternative scenarios for methane escape. J Geophys Res, 2010, 115: E12018

60 Bell J M, Bougher S W, Waite Jr J H, et al. Simulating the one-dimensional structure of Titan's upper atmosphere: 3. Mechanisms determining methane escape. J Geophys Res, 2011, 116: E11002

61 Bell J M, Waite Jr J H, Westlake J H, et al. Developing a self-consistent description of Titan's upper atmosphere without hydrodynamic escape. J Geophys Res Space Phys, 2014, 119: 4957-4972

62 Bougher S W, Dickinson R E, Ridley E C, et al. Venus mesosphere and thermosphere. Icarus, 1988, 73: 545-573

63 Bougher S W, Gérard J C, Stewart A I F, et al. The Venus nitric oxide night airglow: Model calculations based on the Venus thermospheric general circulation model. J Geophys Res, 1990, 95: 6271-6284

64 Bougher S W, Borucki W J. Venus $\mathrm{O}_{2}$ visible and IR nightglow: Implications for lower thermosphere dynamics and chemistry. J Geophys Res, 1994, 99: 3759-3776

65 Zhang S, Bougher S W, Alexander M J. The impact of gravity waves on the Venus thermosphere and $\mathrm{O}_{2}$ IR nightglow. J Geophys Res, 1996, 101: 23195-23205

66 Bougher S W, Alexander M J, Mayr H G. Upper atmosphere dynamics: Global circulation and gravity waves. In: Bougher S W, Hunten D M, 
Philips R J, eds. Venus II. Tucson: University of Arizona Press, 1997. 259-292

67 Bougher S W, Engel S, Roble R G, et al. Comparative terrestrial planet thermospheres: 2. Solar cycle variation of global structure and winds at equinox. J Geophys Res, 1999, 104: 16591-16611

68 Bougher S W, Roble R G, Fuller-Rowell T J. Simulations of the upper atmospheres of the terrestrial planets, in Atmospheres in the Solar System: Comparative Aeronomy. In: Mendillo M, Nagy A, Waite H, eds. Geophysical Monograph, Vol. 130. Washington: American Geophysical Union, 2002. 261-288

69 Bougher S, Keating G, Zurek R, et al. Mars global surveyor aerobraking: Atmospheric trends and model interpretation. Adv Space Res, 1999, 23: 1887-1897

70 Bougher S W, Engel S, Roble R G, et al. Comparative terrestrial planet thermospheres: 3. Solar cycle variation of global structure and winds at solstices. J Geophys Res, 2000, 105: 17669-17692

71 Bougher S W. MGS radio science electron density profiles: Interannual variability and implications for the Martian neutral atmosphere. J Geophys Res, 2004, 109: E03010

72 Bougher S W, Bell J M, Murphy J R, et al. Polar warming in the Mars thermosphere: Seasonal variations owing to changing insolation and dust distributions. Geophys Res Lett, 2006, 33: L02203

73 Bell J M, Bougher S W, Murphy J R. Vertical dust mixing and the interannual variations in the Mars thermosphere. J Geophys Res, 2007, 112: E12002

74 López-Valverde M A, Edwards D P, López-Puertas M, et al. Non-local thermodynamic equilibrium in general circulation models of the Martian atmosphere 1. Effects of the local thermodynamic equilibrium approximation on thermal cooling and solar heating. J Geophys Res, 1998, 103: 16799-16812

75 Bougher S W, Pawlowski D, Bell J M, et al. Mars Global Ionosphere-Thermosphere Model: Solar cycle, seasonal, and diurnal variations of the Mars upper atmosphere. J Geophys Res Planets, 2015, 120: 311-342

76 Ren Z P, Liu Y B, Wan W X, et al. Design of the Mars Thermosphere-Ionosphere Model (in Chinese). Annual Meeting of Chinese Geoscience Union (CGU), 2017 [任志鹏, 刘耘博, 万卫星, 等. 火星热层电离层耦合模式设计. 2017年中国地球科学联合学术年会, 2017]

77 Achilleos N, Miller S, Tennyson J, et al. JIM: A time-dependent, three-dimensional model of Jupiter's thermosphere and ionosphere. J Geophys Res, 1998, 103: 20089-20112

78 Bougher S W. Jupiter Thermospheric General Circulation Model (JTGCM): Global structure and dynamics driven by auroral and Joule heating. J Geophys Res, 2005, 110: E04008

79 Majeed T, Waite Jr J H, Bougher S W, et al. Processes of equatorial thermal structure at Jupiter: An analysis of the Galileo temperature profile with a three-dimensional model. J Geophys Res, 2005, 110: E12007

80 Majeed T, Waite J H, Bougher S W, et al. Processes of auroral thermal structure at Jupiter: Analysis of multispectral temperature observations with the Jupiter Thermosphere General Circulation Model. J Geophys Res, 2009, 114: E07005

81 Yelle R V, Miller S. Jupiter's thermosphere and ionosphere. In: Bagenal F, Dowling T, McKinnon W, eds. Jupiter: The Planet, Satellites, and Magnetosphere. New York: Cambridge University Press, 2004. 185- 218

82 Müller-Wodarg I, Mendillo M, Yelle R, et al. A global circulation model of Saturn's thermosphere. Icarus, 2006, 180: 147-160

83 Müller-Wodarg I C F, Yelle R V, Mendillo M, et al. The thermosphere of Titan simulated by a global three-dimensional time-dependent model. J Geophys Res, 2000, 105: 20833-20856

84 Bell J M, Westlake J, Waite J H. Simulating the time-dependent response of Titan's upper atmosphere to magnetospheric forcing. Geophys Res Lett, 2011, 38: L06202

85 Bougher S W, Blelly P L, Combi M, et al. Neutral upper atmosphere and ionosphere modeling. Space Sci Rev, 2008, 139: 107-141 


\title{
Upper atmosphere modeling: From Earth to Planet
}

\author{
Zhipeng Ren ${ }^{1,2,3,4}$ \\ ${ }^{1}$ Key Laboratory of Earth and Planetary Physics, Institute of Geology and Geophysics, Chinese Academy of Sciences, Beijing 100029, China; \\ ${ }^{2}$ Beijing National Observatory of Space Environment, Institute of Geology and Geophysics, Chinese Academy of Sciences, Beijing 100029, China; \\ 3 Innovation Academy for Earth Science, Chinese Academy of Sciences, Beijing 100029, China; \\ ${ }^{4}$ College of Earth and Planetary Sciences, University of Chinese Academy of Sciences, Beijing 100049, China \\ E-mail: zpren@mail.iggcas.ac.cn
}

The upper atmosphere is the main area of human space activities. It is affected by the neutral atmospheric process, the space plasma process and the coupling between the atmosphere and the space environment. The upper atmosphere is an interdisciplinary research field of atmospheric science and space science.

Studying the process and characteristics of upper atmosphere and revealing their formation mechanism are the key tasks in space physics and space weather research. Observations always have the limitations of type and spatio-temporal coverage. Therefore, the numerical models play a more important role to reproduce various physical and chemical processes of the upper atmosphere comprehensively. They have exhibited unique advantages and been one of the hot spots in the community.

The upper atmosphere cannot be simply regarded as an extension of the middle and lower atmosphere physically. The numerical model of the upper atmosphere must take into account the limitations induced by the characteristics of the upper atmosphere in model design, coordinate system selection and model algorithm. Therefore, the model development of the upper atmosphere has experienced a long time.

In the past decades, modeling of the Earth's upper atmosphere has made great progress. The community has successfully developed several self-consistent numerical models of the upper atmosphere such as TIEGCM, CTIPe, GCITEMIGGCAS, and etc. These models have made great contributions in the research of the Earth's upper atmosphere.

Although the upper atmosphere of the Earth and other planets show obvious differences, the upper atmosphere numerical models of the Earth and other planets closely relate with each other. Most models of other planets were developed on the basis of the Earth's models. Great progress has been made in the numerical modeling and corresponding research of the planetary upper atmosphere.

Due to the lack of understanding of the planetary upper atmosphere at present, comparing with the numerical model of the Earth's upper atmosphere, there are many problems in the numerical model of the planetary upper atmosphere, such as the relatively simple physical process, poor ability to reproduce the real planetary upper atmosphere, insufficient selfconsistency of the model, dependence on the input from empirical model or observation. In general, the development of the numerical model of the planetary upper atmosphere is relatively lagging behind. The numerical models of the planetary upper atmosphere need further development.

This review focuses on the upper atmosphere numerical models of the Earth and planets. We will systematically introduce the development history and performance of the corresponding main models.

upper atmosphere, general circulation model, ionosphere, space environment, planet, Honghu program

doi: 10.1360/TB-2019-0803 